\title{
Decontamination Factors and Filtration Flux Impact to ARP at Reduced MST Concentration
}

\author{
D. T. Hobbs \\ D. T. Herman \\ M. R. Poirier
}

June 2012

Savannah River National Laboratory Savannah River Nuclear Solutions, LLC Aiken, SC 29808

Prepared for the U.S. Department of Energy under contract number DE-AC09-08SR22470. 
SRNL-STI-2012-00299

Revision 0

\section{DISCLAIMER}

This work was prepared under an agreement with and funded by the U.S. Government. Neither the U.S. Government or its employees, nor any of its contractors, subcontractors or their employees, makes any express or implied:

1. warranty or assumes any legal liability for the accuracy, completeness, or for the use or results of such use of any information, product, or process disclosed; or

2. representation that such use or results of such use would not infringe privately owned rights; or

3. endorsement or recommendation of any specifically identified commercial product, process, or service.

Any views and opinions of authors expressed in this work do not necessarily state or reflect those of the United States Government, or its contractors, or subcontractors.

Printed in the United States of America

Prepared for

U.S. Department of Energy 
Keywords: tank waste, plutonium, neptunium, uranium, strontium, ion exchange

Retention: Permanent

\title{
Decontamination Factors and Filtration Flux Impact to ARP at Reduced MST Concentration
}

\author{
D. T. Hobbs \\ D. T. Herman \\ M. R. Poirier
}

June 2012

Savannah River National Laboratory Savannah River Nuclear Solutions, LLC Aiken, SC 29808

Prepared for the U.S. Department of Energy under contract number DE-AC09-08SR22470. 


\section{REVIEWS AND APPROVALS}

AUTHORS:

D. T. Hobbs, Separations and Actinide Science Programs

Date

D. T. Herman, Advanced Characterization and Process Research

Date

M. R. Poirier, Advanced Characterization and Process Research

Date

TECHNICAL REVIEW:

C. A. Nash, Advanced Characterization and Process Research

Date

APPROVAL:

S. D. Fink, Manager

Date

Separations and Actinide Science Programs

Date

S.L. Marra, Manager

Environmental \& Chemical Process Technology Research Programs

D. J. Martin, Manager

Date

Tank Farm and Closure Engineering 
SRNL-STI-2012-00299

Revision 0

\section{EXECUTIVE SUMMARY}

Tank Farm and Closure Engineering is evaluating changes to the Actinide Removal Process facility operations to decrease the MST concentration from $0.4 \mathrm{~g} / \mathrm{L}$ to $0.2 \mathrm{~g} / \mathrm{L}$ and the contact time from 12 hours to between 6 and 8 hours. For this evaluation, SRNL reviewed previous datasets investigating the performance of MST at $0.2 \mathrm{~g} / \mathrm{L}$ in salt solutions ranging from 4.5 to $7.5 \mathrm{M}$ in sodium concentration. In general, reducing the MST concentration from 0.4 to $0.2 \mathrm{~g} / \mathrm{L}$ and increasing the ionic strength from 4.5 to $7.5 \mathrm{M}$ in sodium concentration will decrease the measured decontamination factors for plutonium, neptunium, uranium and strontium. The decontamination factors as well as single standard deviation values for each sorbate are reported. These values are applicable within the sorbate and sodium concentrations used in the experimental measurements.

Decreasing the MST concentration in the ARP from $0.4 \mathrm{~g} / \mathrm{L}$ to $0.2 \mathrm{~g} / \mathrm{L}$ will produce an increase in the filter flux, and could lead to longer operating times between filter cleaning. The increase in flux is a function of a number of operating parameters, and is difficult to quantify. However, it is estimated that the reduction in MST could result in a reduction of filtration time of up to $20 \%$. 


\section{TABLE OF CONTENTS}

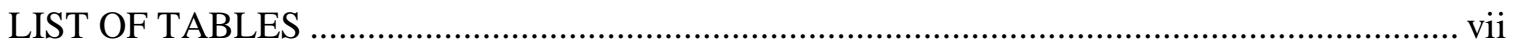

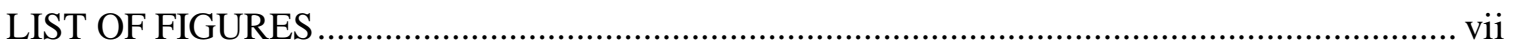

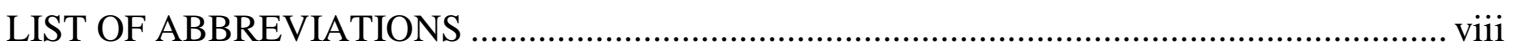

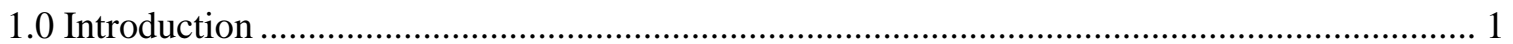

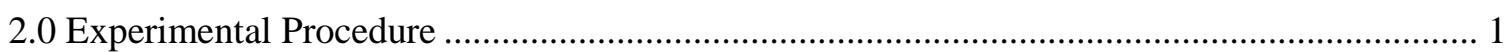

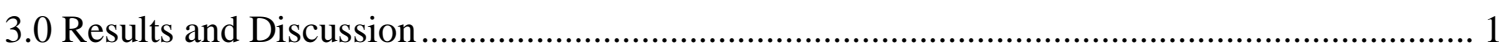

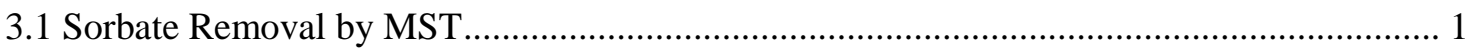

3.2 Filtration Performance at Reduced MST Concentration.................................................... 10

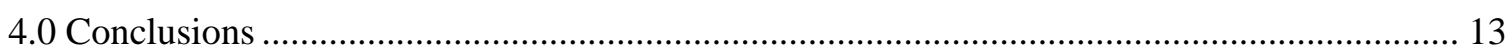

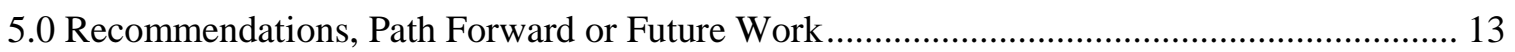

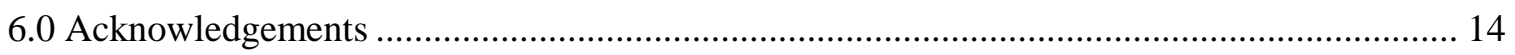

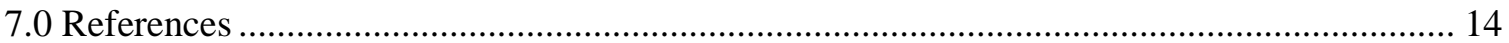


SRNL-STI-2012-00299

Revision 0

\section{LIST OF TABLES}

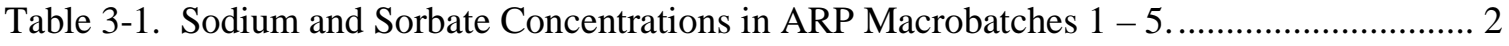

Table 3-2. Initial Sorbate Concentrations in MST Performance Testing ...................................... 3

Table 3-3. Average plutonium DF values upon contact with 0.2 and $0.4 \mathrm{~g} / \mathrm{L}$ MST. Number in the bracket identifying the data set is the reference number from which the experimental data was obtained.

Table 3-4. Average uranium DF values upon contact with 0.2 and $0.4 \mathrm{~g} / \mathrm{L} \mathrm{MST}$. Number in the bracket identifying the data set is the reference number from which the experimental data was obtained.

Table 3-5. Average neptunium DF values upon contact with 0.2 and $0.4 \mathrm{~g} / \mathrm{L} \mathrm{MST}$. Number in the bracket identifying the data set is the reference number from which the experimental data was obtained.

Table 3-6. Average strontium DF values upon contact with 0.2 and $0.4 \mathrm{~g} / \mathrm{L} \mathrm{MST}$. Number in the bracket identifying the data set is the reference number from which the experimental data was obtained.

Table 3-7. Minimum DF Values and Single Standard Deviation for Plutonium, Uranium, Neptunium and Strontium in Alkaline Salt Solutions of Varying Ionic Strength Contacted with $0.2 \mathrm{~g} / \mathrm{L}$ or $0.4 \mathrm{~g} / \mathrm{L}$ MST

\section{LIST OF FIGURES}

Figure 3-1. Comparing Filter Flux Prediction with ARP Data................................................... 11

Figure 3-2. Comparing Corrected Filter Flux Prediction with ARP Data................................... 12 


\section{LIST OF ABBREVIATIONS}

$\begin{array}{ll}\text { ARP } & \text { Actinide Removal Process } \\ \text { DF } & \text { decontamination factor } \\ \text { MB } & \text { macrobatch } \\ \text { MST } & \text { monosodium titanate } \\ \text { MCU } & \text { Modular Caustic Side Solvent Extraction Unit } \\ \text { SRNL } & \text { Savannah River National Laboratory } \\ \text { TF\&CE } & \text { Tank Farm and Closure Engineering } \\ \text { TTR } & \text { Technical Task Request } \\ \text { TMP } & \text { transmembrane pressure }\end{array}$




\subsection{Introduction}

Monosodium titanate (MST) is used in the Actinide Removal Process (ARP) facility to remove ${ }^{90} \mathrm{Sr}$ and alpha-emitting radionuclides, principally the radioisotopes of plutonium, neptunium, and uranium. The current ARP operation adds MST at a concentration of $0.4 \mathrm{~g} / \mathrm{L}$ to each batch of waste processed in the 241-96H strike tanks. The reaction suspension is mixed for 12 hours and then transferred to the 512-S facility for filtration to concentrate the MST solids. The clarified filtrate from the crossflow filters transfers into the Modular Caustic Side Solvent Extraction Unit (MCU) for removal of cesium.

Tank Farm and Closure Engineering (TF\&CE) are evaluating modifications to the ARP flowsheet to increase throughput. These modifications include reducing the MST concentration and reducing the strike time to eight hours or less. To support this evaluation, TF\&CE requested that SRNL determine decontamination factors (DF) for Pu, Np, U and Sr at an MST concentration of $0.2 \mathrm{~g} / \mathrm{L}$ with contact times of 6 and 8 hours in salt solutions having sodium concentrations ranging from $4.5 \mathrm{M}$ to $7.5 \mathrm{M} .{ }^{1}$ SRNL was also requested to determine the effect of the reduced MST concentration on the 512-S crossflow filtration rate. This report summarizes the findings of these evaluations.

\subsection{Experimental Procedure}

No experimental work was performed in support of the Technical Task Request (TTR). ${ }^{1}$

\subsection{Results and Discussion}

\subsection{Sorbate Removal by MST}

The DF for a sorbate is the ratio of the initial solution concentration and the concentration measured at some time after the introduction of the MST. Parameters that influence DF values include the initial sorbate concentration, the ionic strength or sodium ion concentration of the solution, the MST concentration, and the temperature. Temperature is controlled in the ARP process strike tanks at $25 \pm 5{ }^{\circ} \mathrm{C}$. ${ }^{2}$ Previous testing has shown that this small temperature range has minimal influence on MST performance. ${ }^{3}$ Therefore, this evaluation did not consider the effect of temperature.

The ARP initiated radioactive operations in April 2008 and has processed more than 2.8 million gallons of liquid waste through March of 2012. The liquid phase processed through ARP is staged as large, well-characterized batches in Tank $49 \mathrm{H}$. Table 3-1 provides a summary of sodium, strontium and actinide concentrations for the five macrobatches of waste that have been assembled and processed or will be processed in ARP. ${ }^{3-8}$

The target sodium concentration for the feed solution to ARP was originally set at $5.6 \mathrm{M}$. However, the ARP facility has moved to processing waste solutions having sodium concentrations in excess of $6.0 \mathrm{M}$. Previous testing has shown that MST performance as measured by the decontamination factor decreases with increasing sodium concentration and lower MST concentration. ${ }^{9-126}$ Thus, the DF values for $\mathrm{Sr}, \mathrm{Pu}, \mathrm{Np}$ and $\mathrm{U}$ would be expected to be lower upon processing waste solutions with higher sodium concentrations.

Currently, the MST strike tanks in ARP are charged with MST at a concentration of $0.4 \mathrm{~g} / \mathrm{L}$. TF\&CE requested SRNL to provide recommended DF values for each of the sorbates upon decreasing the MST concentration by $50 \%$ to $0.2 \mathrm{~g} / \mathrm{L}$. As shown previously, sorbate removal (i.e., DF value) decreases upon a decrease in the MST concentration. ${ }^{9-12}$ 
SRNL-STI-2012-00299

Revision 0

Table 3-1. Sodium and Sorbate Concentrations in ARP Macrobatches 1 - 5.

\begin{tabular}{|c|c|c|c|c|c|}
\hline & MB1 & MB2 & MB3 & MB4 & MB5 \\
\hline Na (M) & 5.26 & 5.55 & 6.73 & 6.57 & 6.25 \\
\hline $\begin{array}{c}\text { Total Sr } \\
\text { (ug/L) }\end{array}$ & $\leq 2.77 \mathrm{E}+01$ & $\leq 7.5 \mathrm{E}+01$ & $\leq 3.49 \mathrm{E}+01$ & $\leq 4.61 \mathrm{E}+01$ & $\leq 2.42 \mathrm{E}+01$ \\
\hline $\begin{array}{c}\text { Total Pu } \\
\text { (ug/L) }\end{array}$ & $1.08 \mathrm{E}+01$ & $4.77 \mathrm{E}+00$ & $9.29 \mathrm{E}+00$ & $3.57 \mathrm{E}+01$ & $2.20 \mathrm{E}+01$ \\
\hline $\begin{array}{c}\text { Np-237 } \\
\text { (ug/L) }\end{array}$ & $2.54 \mathrm{E}+01$ & $\leq 5.01 \mathrm{E}+01$ & $2.94 \mathrm{E}+01$ & $2.16 \mathrm{E}+01$ & $4.81 \mathrm{E}+00$ \\
\hline $\begin{array}{c}\text { Total U } \\
\text { (ug/L) }\end{array}$ & $1.78 \mathrm{E}+03$ & $5.30 \mathrm{E}+03$ & $1.82 \mathrm{E}+04$ & $2.63 \mathrm{E}+04$ & $1.11 \mathrm{E}+04$ \\
\hline
\end{tabular}

There have been several experimental data sets that have measured the performance of MST at a concentration of $0.2 \mathrm{~g} / \mathrm{L}$ with salt solutions having sodium concentrations ranging between $4.5 \mathrm{M}$ and $7.5 \mathrm{M}^{9-13}$ These tests were carried out at $25 \pm 3{ }^{\circ} \mathrm{C}$, which is very similar to the temperature range specified for ARP operations. Table 3-2 provides the initial sorbate and sodium concentrations for each of the test solutions used in these experiments. Within analytical uncertainty, the strontium, plutonium, neptunium, and uranium concentrations in the test solutions are equal to or exceed those in the waste solutions processed to date in ARP. Note that the uranium is the only sorbate in any of the ARP macrobatch feed solutions that is at or close to the maximum soluble concentration in the simulant and tank waste solutions tested in the laboratory for MST performance. Thus, the results reported in this document are suitable for estimating operating performance in ARP. If future ARP feed solutions have sorbate concentrations outside of the ranges reported in Tables 3-1 and 3-2, the expected MST performance should be reassessed.

Note also, that there are considerable differences among the three datasets in the range of sorbate concentrations. The ratio between the minimum and maximum sorbate concentrations vary from 1.67 for plutonium (lowest variation), 3.33 for uranium, 38.1 for strontium and 270 for neptunium (highest variation). Given this wide of range in sorbate concentrations, particularly for neptunium and strontium, DF values can differ considerably for solutions that have the same ionic strength (i.e., same sodium concentration), but different initial sorbate concentrations.

The TTR requested that SRNL provide DF values for 6 and 8-hour contact times. However, the available datasets have much more data at a 4-hour contact time and very little data at the 6-hour contact time. With concurrence from TF\&CE, we evaluated DF values for each of the sorbates at 4-hour, 6-hour and 8-hour contact times and compared these values to that determined at 12hours, which represents the current contact time in the ARP facility. 
SRNL-STI-2012-00299

Revision 0

Table 3-2. Initial Sorbate Concentrations in MST Performance Testing

\begin{tabular}{|c|c|c|c|c|c||}
\hline [Na] M & {$[\mathbf{P u}] \mathbf{~ u g} / \mathbf{L}$} & {$[\mathbf{N p}] \mathbf{~ u g} / \mathbf{L}$} & {$[\mathbf{U}] \mathbf{~ u g} / \mathbf{L}$} & [Sr] ug/L & Reference \\
\hline 4.5 & 168 & 21,000 & 14,800 & 63.6 & 9 \\
\hline 4.5 & 63.5 & 406 & 9,020 & 90.1 & 12 \\
\hline 5.4 & 114 & 457 & 9260 & 1,680 & 13 \\
\hline 5.6 & 191 & 405 & 9,010 & 90.5 & 10 \\
\hline 5.6 & 254 & 129 & 10,200 & 1,580 & 11 \\
\hline 5.6 & 218 & 461 & 9,550 & 484 & 11 \\
\hline 6.8 & 142 & 487 & 4,010 & 2,420 & 13 \\
\hline 7.5 & 280 & 35,000 & 24,600 & 106 & 9 \\
\hline
\end{tabular}

Tables 3-3 through 3-6 provides a listing of measured and calculated DF values and single standard deviation at MST concentrations of 0.2 and $0.4 \mathrm{~g} / \mathrm{L}$. Calculated DF values were derived for selected contact times by fitting the DF versus contact time data to either a linear or second order polynomial function for each data set. These graphs and the mathematical equations are provided in the Appendix for each sorbate.

With the exception of uranium, the general trend is that the DF value decreases with a decrease in the MST concentration, a decrease in the contact time and an increase in the sodium concentration of the waste solution. For example, four of the datasets (Phase IV, Phase V and two set from Phase II mMST) measured MST performance at both $0.2 \mathrm{~g} / \mathrm{L}$ and $0.4 \mathrm{~g} / \mathrm{L}$ and, thus, provide the best comparison for evaluating the influence of MST concentration. Comparing the DF values at each of the contact times within each data set indicated that decreasing the MST concentration from 0.4 to $0.2 \mathrm{~g} / \mathrm{L}$ decreased the DF value by a factor ranging from 1.6 to 2.6, with a majority of the results very close to a value of two. A decrease in the DF value by a factor of two would be expected assuming no other parameters (e.g., changes in other sorbate concentrations) are influencing plutonium removal.

For plutonium, shorter contact times resulted in reduced DF values for all but one of the data sets. For example, in the Phase $\mathrm{IV}^{6}$ and Phase $\mathrm{V}^{4}$ datasets, the plutonium DF value at a contact time of 4 hours is about $60 \%$ of that measured or calculated to be at 12 hours. At a contact time of 8 hours, the DF value is about $82 \%$ of that at 12 hours for single contacts with either 0.2 or $0.4 \mathrm{~g} / \mathrm{L}$ MST. In the Phase II mMST data sets with simulated and actual tank wastes, the impact of reduced contact times proved smaller at the 4-hour and 8-hour contact time DF values measured about $80 \%$ to $100 \%$ of the 12 -hour values at both MST concentrations.

Comparison of the DF values at each of the contact times for the salt solutions having increasing sodium concentrations revealed that the plutonium DF value at $5.6 \mathrm{M} \mathrm{Na}$ is about a factor of two lower than that measured at $4.5 \mathrm{M} \mathrm{Na}$. For a 7.5 M Na salt solution the plutonium DF value is decreased by a factor of about four compared to that measured in a $4.5 \mathrm{M}$ Na salt solution. 
SRNL-STI-2012-00299

Revision 0

Table 3-3. Average plutonium DF values upon contact with 0.2 and $0.4 \mathrm{~g} / \mathrm{L}$ MST. Number in the bracket identifying the data set is the reference number from which the experimental data was obtained.

\begin{tabular}{|c|c|c|c|c|c|c|}
\hline \multirow[b]{2}{*}{ Data Set } & \multirow[b]{2}{*}{ [Na] M } & \multirow{2}{*}{$\begin{array}{c}\text { Initial } \\
{[\mathrm{Pu}] \text { ug/L }}\end{array}$} & \multicolumn{4}{|c|}{ Average Pu DF with 0.2 g/L MST } \\
\hline & & & 4-hour & 6-hour & 8-hour & 12-hour \\
\hline Phase III [3] & 4.5 & $1.68 \mathrm{E}+02$ & $3.19 \mathrm{E}+00$ & nd & $7.10 E+01$ & nd \\
\hline Phase IV [6] & 4.5 & $6.35 \mathrm{E}+01$ & $5.51 \mathrm{E}+00$ & nd & $7.25 \mathrm{E}+00$ & $9.03 \mathrm{E}+00$ \\
\hline Phase V [4] & 5.6 & $1.93 \mathrm{E}+02$ & $3.21 \mathrm{E}+00$ & nd & $4.56 \mathrm{E}+00$ & $5.31 \mathrm{E}+00$ \\
\hline Phase II mMST [5] & 5.6 & $2.00 \mathrm{E}+02$ & $2.83 \mathrm{E}+00$ & nd & $2.95 \mathrm{E}+00$ & $3.21 \mathrm{E}+00$ \\
\hline Phase II mMST [5] & 5.6 & $2.54 \mathrm{E}+02$ & $2.99 \mathrm{E}+00$ & $1.86 \mathrm{E}+00$ & $2.54 \mathrm{E}+00$ & $2.35 \mathrm{E}+00$ \\
\hline Phase III [3] & 7.5 & $2.80 \mathrm{E}+02$ & $1.41 \mathrm{E}+00$ & nd & $1.91 \mathrm{E}+00$ & $2.29 \mathrm{E}+00$ \\
\hline \multirow[b]{2}{*}{ Data Set } & \multirow[b]{2}{*}{ [Na] $\mathbf{M}$} & \multirow{2}{*}{$\begin{array}{c}\text { Initial } \\
{[\mathbf{P u}] \text { ug/L }}\end{array}$} & \multicolumn{4}{|c|}{$\begin{array}{l}\text { Single Standard Deviation of Pu DF with } 0.2 \mathrm{~g} / \mathrm{L} \\
\text { MST }\end{array}$} \\
\hline & & & 4-hour & 6-hour & 8-hour & 12-hour \\
\hline Phase III [3] & 4.5 & $1.68 \mathrm{E}+02$ & 5.49E-02 & ind & $9.87 \mathrm{E}+00$ & ind \\
\hline Phase IV [6] & 4.5 & $6.35 \mathrm{E}+01$ & 4.47E-01 & ind & $5.42 \mathrm{E}-02$ & ind \\
\hline Phase V [4] & 5.6 & $1.93 \mathrm{E}+02$ & 5.69E-02 & ind & 3.67E-01 & ind \\
\hline Phase II mMST [5] & 5.6 & $2.00 \mathrm{E}+02$ & $2.00 \mathrm{E}-01$ & ind & ind & 2.40E-01 \\
\hline Phase II mMST [5] & 5.6 & $2.54 \mathrm{E}+02$ & 2.12E-01 & 1.36E-01 & ind & $1.79 \mathrm{E}-01$ \\
\hline Phase III [3] & 7.5 & $2.80 \mathrm{E}+02$ & 6.30E-02 & ind & 2.17E-01 & ind \\
\hline \multirow[b]{2}{*}{ Data Set } & \multirow[b]{2}{*}{ [Na] M } & \multirow{2}{*}{$\begin{array}{c}\text { Initial } \\
{[\mathrm{Pu}] \mathrm{ug} / \mathrm{L}}\end{array}$} & \multicolumn{4}{|c|}{ Average Pu DF with 0.4 g/L MST } \\
\hline & & & 4-hour & 6-hour & 8-hour & 12-hour \\
\hline Phase IV [6] & 4.5 & $6.35 \mathrm{E}+01$ & $1.15 \mathrm{E}+01$ & nd & $1.42 \mathrm{E}+01$ & $1.82 \mathrm{E}+01$ \\
\hline Phase V [4] & 5.6 & $1.93 \mathrm{E}+02$ & $6.48 \mathrm{E}+00$ & nd & $7.64 \mathrm{E}+00$ & $9.67 \mathrm{E}+00$ \\
\hline Phase II mMST [5] & 5.6 & $2.00 \mathrm{E}+02$ & $5.06 \mathrm{E}+00$ & $4.78 \mathrm{E}+00$ & $5.40 \mathrm{E}+00$ & $6.45 \mathrm{E}+00$ \\
\hline Phase II mMST [5] & 5.6 & $2.54 \mathrm{E}+02$ & $4.79 \mathrm{E}+00$ & nd & $5.45 \mathrm{E}+00$ & $6.25 \mathrm{E}+00$ \\
\hline Engineered MST [7] & 5.4 & $1.14 \mathrm{E}+02$ & nd & $2.62 \mathrm{E}+00$ & nd & nd \\
\hline Engineered MST [7] & 6.8 & $1.42 \mathrm{E}+02$ & nd & $2.51 \mathrm{E}+00$ & nd & nd \\
\hline \multirow[b]{2}{*}{ Data Set } & \multirow[b]{2}{*}{ [Na] M } & \multirow{2}{*}{$\begin{array}{c}\text { Initial } \\
{[\mathrm{Pu}] \mathrm{ug} / \mathrm{L}}\end{array}$} & \multicolumn{4}{|c|}{$\begin{array}{l}\text { Single Standard Deviation of Pu DF with } 0.4 \mathrm{~g} / \mathrm{L} \\
\text { MST }\end{array}$} \\
\hline & & & 4-hour & ind & 8-hour & 12-hour \\
\hline Phase IV [6] & 4.5 & $6.35 \mathrm{E}+01$ & 2.77E-01 & ind & $1.59 \mathrm{E}+00$ & ind \\
\hline Phase V [4] & 5.6 & $1.93 \mathrm{E}+02$ & 4.36E-01 & ind & $1.57 \mathrm{E}+00$ & ind \\
\hline Phase II mMST [5] & 5.6 & $2.00 \mathrm{E}+02$ & $0.00 \mathrm{E}+00$ & $0.00 \mathrm{E}+00$ & ind & $0.00 \mathrm{E}+00$ \\
\hline Phase II mMST [5] & 5.6 & $2.54 \mathrm{E}+02$ & 3.90E-01 & - & ind & 4.43E-01 \\
\hline Engineered MST [7] & 5.4 & $1.14 \mathrm{E}+02$ & ind & 2.39E-01 & ind & ind \\
\hline Engineered MST [7] & 6.8 & $1.42 \mathrm{E}+02$ & ind & 2.65E-01 & ind & ind \\
\hline \multicolumn{3}{|c|}{ red number signifies calculated value } & \multicolumn{2}{|c|}{ nd $=$ not determined } & & \\
\hline \multicolumn{7}{|c|}{$\begin{array}{l}\text { "ind" signifies that the standard deviation is indeterminate due to one of the following reasons; the DF was not } \\
\text { determined experimentally, the experimental DF result was not a quantifiable value, or that the DF is a } \\
\text { calculated value. }\end{array}$} \\
\hline \multicolumn{7}{|c|}{$\begin{array}{l}\text { Italicized DF value at a contact time of } 8 \text { hours in the Phase III dataset with the } 4.5 \mathrm{M} \text { Na solution is not } \\
\text { consistent with the 4-hour result as well as results from other datasets (e.g., Phase IV). Thus, the 8-hour result } \\
\text { and a corresponding calculated result for a 12-hour contact time were not used in the evaluation for this report. }\end{array}$} \\
\hline
\end{tabular}


Table 3-4. Average uranium DF values upon contact with 0.2 and 0.4 g/L MST. Number in the bracket identifying the data set is the reference number from which the experimental data was obtained.

\begin{tabular}{|c|c|c|c|c|c|c|}
\hline \multirow[b]{2}{*}{ Data Set } & \multirow[b]{2}{*}{ [Na] M } & \multirow{2}{*}{$\begin{array}{c}\text { Initial [U] } \\
\text { ug/L }\end{array}$} & \multicolumn{4}{|c|}{ Average U DF with $0.2 \mathrm{~g} / \mathrm{L}$} \\
\hline & & & 4-hour & & 8-hour & 12-hour \\
\hline Phase III [3] & 4.5 & $7.39 \mathrm{E}+03$ & $1.23 \mathrm{E}+00$ & nd & $1.23 \mathrm{E}+00$ & $1.23 \mathrm{E}+00$ \\
\hline Phase IV [6] & 4.5 & $9.02 \mathrm{E}+03$ & $1.11 \mathrm{E}+00$ & nd & $1.13 \mathrm{E}+00$ & $1.14 \mathrm{E}+00$ \\
\hline Phase V [4] & 5.6 & $8.98 \mathrm{E}+03$ & $1.12 \mathrm{E}+00$ & nd & $1.04 \mathrm{E}+00$ & $1.24 \mathrm{E}+00$ \\
\hline Phase II mMST [5] & 5.6 & $1.04 \mathrm{E}+04$ & nd & nd & nd & nd \\
\hline Phase II mMST [5] & 5.6 & $1.02 \mathrm{E}+04$ & nd & nd & nd & nd \\
\hline Phase III [3] & 7.5 & $2.46 \mathrm{E}+04$ & $1.02 \mathrm{E}+00$ & nd & $1.04 \mathrm{E}+00$ & $1.18 \mathrm{E}+00$ \\
\hline \multirow[b]{2}{*}{ Data Set } & \multirow[b]{2}{*}{ [Na] M } & \multirow{2}{*}{$\begin{array}{c}\text { Initial [U] } \\
\text { ug/L }\end{array}$} & \multicolumn{4}{|c|}{ Single Standard Deviation of U DF with $0.2 \mathrm{~g} / \mathrm{L} \mathrm{MST}$} \\
\hline & & & 4-hour & 6-hour & 8-hour & 12-hour \\
\hline Phase III [3] & 4.5 & $7.39 \mathrm{E}+03$ & $0.00 \mathrm{E}+00$ & ind & - & ind \\
\hline Phase IV [6] & 4.5 & $9.02 \mathrm{E}+03$ & $0.00 \mathrm{E}+00$ & ind & 2.96E-02 & ind \\
\hline Phase V [4] & 5.6 & $8.98 \mathrm{E}+03$ & 3.11E-02 & ind & 1.59E-02 & ind \\
\hline Phase II mMST [5] & 5.6 & $1.04 \mathrm{E}+04$ & ind & ind & ind & ind \\
\hline Phase II mMST [5] & 5.6 & $1.02 \mathrm{E}+04$ & ind & ind & ind & ind \\
\hline Phase III [3] & 7.5 & $2.46 \mathrm{E}+04$ & $0.00 \mathrm{E}+00$ & ind & $1.47 \mathrm{E}-02$ & ind \\
\hline \multirow[b]{2}{*}{ Data Set } & \multirow[b]{2}{*}{ [Na] M } & \multirow{2}{*}{$\begin{array}{c}\text { Initial [U] } \\
\text { ug/L }\end{array}$} & \multicolumn{4}{|c|}{ Average U DF with $0.4 \mathrm{~g} / \mathrm{L}$} \\
\hline & & & 4-hour & nd & 8-hour & 12-hour \\
\hline Phase IV [6] & 4.5 & $9.02 \mathrm{E}+03$ & $1.24 \mathrm{E}+00$ & nd & $1.24 \mathrm{E}+00$ & $1.28 \mathrm{E}+00$ \\
\hline Phase V [4] & 5.6 & $8.98 \mathrm{E}+03$ & $1.04 \mathrm{E}+00$ & nd & $1.22 \mathrm{E}+00$ & $1.20 \mathrm{E}+00$ \\
\hline Phase II mMST [5] & 5.6 & $1.04 \mathrm{E}+04$ & nd & nd & nd & nd \\
\hline Phase II mMST [5] & 5.6 & $1.02 \mathrm{E}+04$ & nd & nd & nd & nd \\
\hline Engr MST [7] & 5.4 & $9.26 \mathrm{E}+03$ & nd & $1.11 \mathrm{E}+00$ & nd & nd \\
\hline Engr MST [7] & 6.8 & $4.01 \mathrm{E}+03$ & nd & $1.06 \mathrm{E}+00$ & nd & nd \\
\hline \multirow[b]{2}{*}{ Data Set } & \multirow[b]{2}{*}{ [Na] M } & \multirow{2}{*}{$\begin{array}{c}\text { Initial [U] } \\
\text { ug/L }\end{array}$} & \multicolumn{4}{|c|}{ Single Standard Deviation of U DF with $0.4 \mathrm{~g} / \mathrm{L}$ MST } \\
\hline & & & 4-hour & 6-hour & 8-hour & 12-hour \\
\hline Phase IV [6] & 4.5 & $9.02 \mathrm{E}+03$ & 2.06E-02 & ind & 1.11E-02 & ind \\
\hline Phase V [4] & 5.6 & $8.98 \mathrm{E}+03$ & 1.53E-01 & ind & 8.17E-02 & ind \\
\hline Phase II mMST [5] & 5.6 & $1.04 \mathrm{E}+04$ & ind & ind & ind & ind \\
\hline Phase II mMST [5] & 5.6 & $1.02 \mathrm{E}+04$ & ind & ind & ind & ind \\
\hline Engr MST [7] & 5.4 & $9.26 \mathrm{E}+03$ & ind & 3.09E-02 & ind & ind \\
\hline Engr MST [7] & 6.8 & $4.01 \mathrm{E}+03$ & ind & 4.19E-02 & ind & ind \\
\hline \multicolumn{3}{|c|}{ red number signifies calculated value } & \multicolumn{2}{|c|}{ nd $=$ not determined } & & \\
\hline \multicolumn{7}{|c|}{$\begin{array}{l}\text { "ind" signifies that the standard deviation is indeterminate due to one of the following reasons; the DF was not } \\
\text { determined experimentally, the experimental DF result was not a quantifiable value, or that the DF is a } \\
\text { calculated value. }\end{array}$} \\
\hline
\end{tabular}


Table 3-5. Average neptunium DF values upon contact with 0.2 and 0.4 g/L MST. Number in the bracket identifying the data set is the reference number from which the experimental data was obtained.

\begin{tabular}{|c|c|c|c|c|c|c|}
\hline \multirow[b]{2}{*}{ Data Set } & \multirow[b]{2}{*}{ [Na] M } & \multirow{2}{*}{$\begin{array}{l}\text { Initial } \\
{[\mathrm{Np}] \mathrm{ug} / \mathrm{L}}\end{array}$} & \multicolumn{4}{|c|}{ Average Np DF with 0.2 g/L MST } \\
\hline & & & 4-hour & 6-hour & 8-hour & 12-hour \\
\hline Phase III [3] & 4.5 & $1.05 \mathrm{E}+04$ & $1.30 \mathrm{E}+00$ & nd & $1.12 \mathrm{E}+00$ & $1.23 \mathrm{E}+00$ \\
\hline Phase IV [6] & 4.5 & $4.06 \mathrm{E}+02$ & $1.69 \mathrm{E}+00$ & nd & $2.06 \mathrm{E}+00$ & $2.37 \mathrm{E}+00$ \\
\hline Phase V [4] & 5.6 & $4.03 \mathrm{E}+02$ & $1.62 \mathrm{E}+00$ & nd & $1.74 \mathrm{E}+00$ & $2.14 \mathrm{E}+00$ \\
\hline Phase II mMST [5] & 5.6 & $4.74 \mathrm{E}+02$ & $1.29 \mathrm{E}+00$ & nd & $1.24 \mathrm{E}+00$ & $1.25 \mathrm{E}+00$ \\
\hline Phase II mMST [5] & 5.6 & $1.29 \mathrm{E}+02$ & $2.87 \mathrm{E}+00$ & $2.55 \mathrm{E}+00$ & $2.96 \mathrm{E}+00$ & $3.24 \mathrm{E}+00$ \\
\hline Phase III [3] & 7.5 & $3.50 \mathrm{E}+04$ & $1.17 \mathrm{E}+00$ & nd & $1.16 \mathrm{E}+00$ & $1.26 \mathrm{E}+00$ \\
\hline \multirow[b]{2}{*}{ Data Set } & \multirow[b]{2}{*}{ [Na] M } & \multirow{2}{*}{$\begin{array}{c}\text { Initial } \\
{[\mathrm{Np}] \mathrm{ug} / \mathrm{L}}\end{array}$} & \multicolumn{4}{|c|}{$\begin{array}{l}\text { Single Standard Deviation of Np DF with } 0.2 \mathrm{~g} / \mathrm{L} \\
\text { MST }\end{array}$} \\
\hline & & & 4-hour & 6-hour & 8-hour & 12-hour \\
\hline Phase III [3] & 4.5 & $1.05 \mathrm{E}+04$ & 1.97E-01 & ind & 1.93E-02 & ind \\
\hline Phase IV [6] & 4.5 & $4.06 \mathrm{E}+02$ & ind & ind & 3.85E-02 & ind \\
\hline Phase V [4] & 5.6 & $4.03 \mathrm{E}+02$ & 5.51E-02 & ind & 4.45E-02 & ind \\
\hline Phase II mMST [5] & 5.6 & $4.74 \mathrm{E}+02$ & 9.80E-02 & ind & ind & $1.00 \mathrm{E}-01$ \\
\hline Phase II mMST [5] & 5.6 & $1.29 \mathrm{E}+02$ & 8.13E-01 & $0.00 \mathrm{E}+00$ & ind & 9.17E-01 \\
\hline Phase III [3] & 7.5 & $3.50 \mathrm{E}+04$ & $0.00 \mathrm{E}+00$ & ind & 4.79E-03 & ind \\
\hline \multirow[b]{2}{*}{ Data Set } & \multirow[b]{2}{*}{ [Na] $\mathbf{M}$} & \multirow{2}{*}{$\begin{array}{l}\text { Initial } \\
{[\mathrm{Np}] \mathrm{ug} / \mathrm{L}}\end{array}$} & \multicolumn{4}{|c|}{ Average Np DF with 0.4 g/L MST } \\
\hline & & & 4-hour & 6-hour & 8-hour & 12-hour \\
\hline Phase IV [6] & 4.5 & $4.06 \mathrm{E}+02$ & $2.24 \mathrm{E}+00$ & nd & $2.73 E+00$ & $3.39 E+00$ \\
\hline Phase V [4] & 5.6 & $4.03 \mathrm{E}+02$ & $1.78 \mathrm{E}+00$ & nd & $2.63 \mathrm{E}+00$ & $3.08 \mathrm{E}+00$ \\
\hline Phase II mMST [5] & 5.6 & $4.74 \mathrm{E}+02$ & $1.27 \mathrm{E}+00$ & $1.31 \mathrm{E}+00$ & $1.33 \mathrm{E}+00$ & $1.40 \mathrm{E}+00$ \\
\hline Phase II mMST [5] & 5.6 & $1.29 \mathrm{E}+02$ & $3.95 \mathrm{E}+00$ & nd & $4.68 \mathrm{E}+00$ & $6.45 \mathrm{E}+00$ \\
\hline Engr MST [7] & 5.4 & $4.57 \mathrm{E}+02$ & nd & $1.14 \mathrm{E}+00$ & nd & nd \\
\hline Engr MST [7] & 6.8 & $4.87 \mathrm{E}+02$ & nd & $2.62 \mathrm{E}+00$ & nd & nd \\
\hline \multirow[b]{2}{*}{ Data Set } & \multirow[b]{2}{*}{ [Na] M } & \multirow{2}{*}{$\begin{array}{c}\text { Initial } \\
{[\mathrm{Np}] \mathrm{ug} / \mathrm{L}}\end{array}$} & \multicolumn{4}{|c|}{$\begin{array}{l}\text { Single Standard Deviation of Np DF with } 0.4 \mathrm{~g} / \mathrm{L} \\
\text { MST }\end{array}$} \\
\hline & & & 4-hour & 6-hour & 8-hour & 12-hour \\
\hline Phase IV [6] & 4.5 & $4.06 \mathrm{E}+02$ & 8.48E-02 & ind & 3.71E-03 & ind \\
\hline Phase V [4] & 5.6 & $4.03 \mathrm{E}+02$ & 2.72E-01 & ind & 2.36E-01 & ind \\
\hline Phase II mMST [5] & 5.6 & $4.74 \mathrm{E}+02$ & $0.00 \mathrm{E}+00$ & $0.00 \mathrm{E}+00$ & ind & $0.00 \mathrm{E}+00$ \\
\hline Phase II mMST [5] & 5.6 & $1.29 \mathrm{E}+02$ & $1.12 \mathrm{E}+00$ & - & ind & $1.83 \mathrm{E}+00$ \\
\hline Engr MST [7] & 5.4 & $4.57 \mathrm{E}+02$ & ind & 1.33E-02 & ind & ind \\
\hline Engr MST [7] & 6.8 & $4.87 \mathrm{E}+02$ & ind & 3.63E-01 & ind & ind \\
\hline \multicolumn{3}{|c|}{ red number signifies calculated value } & \multicolumn{2}{|c|}{ nd $=$ not determined } & & \\
\hline \multicolumn{7}{|c|}{$\begin{array}{l}\text { "ind" signifies that the standard deviation is indeterminate due to one of the following reasons; the DF was not } \\
\text { determined experimentally, the experimental DF result was not a quantifiable value, or that the DF is a calculated } \\
\text { value. }\end{array}$} \\
\hline
\end{tabular}


Table 3-6. Average strontium DF values upon contact with 0.2 and 0.4 g/L MST. Number in the bracket identifying the data set is the reference number from which the experimental data was obtained.

\begin{tabular}{|c|c|c|c|c|c|c|}
\hline \multirow[b]{2}{*}{ Data Set } & \multirow[b]{2}{*}{ [Na] M } & \multirow{2}{*}{$\begin{array}{c}\text { Initial [Sr] } \\
\text { ug/L }\end{array}$} & \multicolumn{4}{|c|}{ Average Sr DF with 0.2 g/L MST } \\
\hline & & & 4-hour & 6-hour & 8-hour & 12-hour \\
\hline Phase III [3] & 4.5 & $6.36 \mathrm{E}+01$ & $1.38 \mathrm{E}+02$ & nd & $1.89 \mathrm{E}+02$ & $2.27 \mathrm{E}+02$ \\
\hline Phase IV [6] & 4.5 & $9.00 \mathrm{E}+01$ & $1.28 \mathrm{E}+02$ & nd & $1.50 \mathrm{E}+02$ & $1.91 \mathrm{E}+02$ \\
\hline Phase V [4] & 5.6 & $9.16 \mathrm{E}+01$ & $2.67 \mathrm{E}+01$ & nd & $3.10 \mathrm{E}+01$ & $3.75 \mathrm{E}+01$ \\
\hline Phase II mMST [5] & 5.6 & $5.69 \mathrm{E}+02$ & $2.17 \mathrm{E}+01$ & nd & $2.20 \mathrm{E}+01$ & $2.48 \mathrm{E}+01$ \\
\hline Phase II mMST [5] & 5.6 & $1.58 \mathrm{E}+03$ & nd & $1.71 \mathrm{E}+01$ & nd & nd \\
\hline Phase III [3] & 7.5 & $1.06 \mathrm{E}+02$ & $5.67 \mathrm{E}+01$ & nd & $6.91 \mathrm{E}+01$ & $7.97 \mathrm{E}+01$ \\
\hline \multirow[b]{2}{*}{ Data Set } & \multirow[b]{2}{*}{ [Na] M } & \multirow{2}{*}{$\begin{array}{l}\text { Initial [Sr] } \\
\text { ug/L }\end{array}$} & \multicolumn{4}{|c|}{$\begin{array}{l}\text { Single Standard Deviation of Sr DF with } 0.2 \mathrm{~g} / \mathrm{L} \\
\text { MST }\end{array}$} \\
\hline & & & 4-hour & 6-hour & 8-hour & 12-hour \\
\hline Phase III [3] & 4.5 & $6.36 \mathrm{E}+01$ & $2.59 \mathrm{E}+01$ & ind & $4.89 \mathrm{E}+00$ & ind \\
\hline Phase IV [6] & 4.5 & $9.00 \mathrm{E}+01$ & $3.31 \mathrm{E}+00$ & ind & 7.89E-01 & ind \\
\hline Phase V [4] & 5.6 & $9.16 \mathrm{E}+01$ & 9.99E-01 & ind & 6.31E-01 & ind \\
\hline Phase II mMST [5] & 5.6 & $5.69 \mathrm{E}+02$ & 6.00E-01 & ind & ind & 7.10E-01 \\
\hline Phase II mMST [5] & 5.6 & $1.58 \mathrm{E}+03$ & ind & $4.85 \mathrm{E}+00$ & ind & ind \\
\hline Phase III [3] & 7.5 & $1.06 \mathrm{E}+02$ & 3.61E-01 & - & $2.77 \mathrm{E}+00$ & ind \\
\hline \multirow[b]{2}{*}{ Data Set } & \multirow[b]{2}{*}{ [Na] $\mathbf{M}$} & \multirow{2}{*}{$\begin{array}{c}\text { Initial [Sr] } \\
\text { ug/L }\end{array}$} & \multicolumn{4}{|c|}{ Average Sr DF with 0.4 g/L MST } \\
\hline & & & 4-hour & 6-hour & 8-hour & 12-hour \\
\hline Phase IV [6] & 4.5 & $9.00 \mathrm{E}+01$ & $3.28 \mathrm{E}+02$ & nd & $3.90 \mathrm{E}+02$ & $4.99 \mathrm{E}+02$ \\
\hline Phase V [4] & 5.6 & $9.16 \mathrm{E}+01$ & $8.79 \mathrm{E}+01$ & nd & $1.10 \mathrm{E}+02$ & $1.32 \mathrm{E}+02$ \\
\hline Phase II mMST [5] & 5.6 & $5.69 \mathrm{E}+02$ & $4.87 \mathrm{E}+01$ & $5.62 \mathrm{E}+01$ & $6.71 \mathrm{E}+01$ & $8.27 \mathrm{E}+01$ \\
\hline Phase II mMST [5] & 5.6 & $1.58 \mathrm{E}+03$ & $>3.04 \mathrm{E}+01$ & $>5.62 \mathrm{E}+01$ & $4.60 \mathrm{E}+01$ & $5.68 \mathrm{E}+01$ \\
\hline Engr MST [7] & 5.4 & $1.68 \mathrm{E}+03$ & nd & $4.60 \mathrm{E}+01$ & nd & nd \\
\hline Engr MST [7] & 6.8 & $2.42 \mathrm{E}+03$ & nd & $1.96 \mathrm{E}+01$ & nd & nd \\
\hline \multirow[b]{2}{*}{ Data Set } & \multirow[b]{2}{*}{ [Na] M } & \multirow{2}{*}{$\begin{array}{c}\text { Initial [Sr] } \\
\text { ug/L }\end{array}$} & \multicolumn{4}{|c|}{$\begin{array}{l}\text { Single Standard Deviation of Sr DF with } 0.4 \mathrm{~g} / \mathrm{L} \\
\text { MST }\end{array}$} \\
\hline & & & 4-hour & 6-hour & 8-hour & 12-hour \\
\hline Phase IV [6] & 4.5 & $9.00 \mathrm{E}+01$ & 8.67E-01 & ind & $2.68 \mathrm{E}+01$ & ind \\
\hline Phase V [4] & 5.6 & $9.16 \mathrm{E}+01$ & $3.17 \mathrm{E}+03$ & ind & $1.72 \mathrm{E}-01$ & ind \\
\hline Phase II mMST [5] & 5.6 & $5.69 \mathrm{E}+02$ & $0.00 \mathrm{E}+00$ & $0.00 \mathrm{E}+00$ & ind & $0.00 \mathrm{E}+00$ \\
\hline Phase II mMST [5] & 5.6 & $1.58 \mathrm{E}+03$ & $0.00 \mathrm{E}+00$ & - & ind & $3.92 \mathrm{E}+00$ \\
\hline Engr MST [7] & 5.4 & $1.68 \mathrm{E}+03$ & ind & $8.65 \mathrm{E}+00$ & ind & ind \\
\hline Engr MST [7] & 6.8 & $2.42 \mathrm{E}+03$ & ind & $1.96 \mathrm{E}+00$ & ind & ind \\
\hline \multicolumn{3}{|c|}{ red number signifies calculated value } & \multicolumn{2}{|c|}{ nd $=$ not determined } & & \\
\hline \multicolumn{7}{|c|}{$\begin{array}{l}\text { "ind" signifies that the standard deviation is indeterminate due to one of the following reasons; the DF was not } \\
\text { determined experimentally, the experimental DF result was not a quantifiable value, or that the DF is a } \\
\text { calculated value. }\end{array}$} \\
\hline
\end{tabular}


For the removal of uranium the measured DF values at the shorter contact times were not statistically different than those measured at 12-hours with either 0.2 or $0.4 \mathrm{~g} / \mathrm{L}$ (see Table 3-4). Furthermore, the DF values were not influenced by Na ion concentrations ranging from $4.5 \mathrm{M}$ to $7.5 \mathrm{M}$ at contact times of between 4 and 12 hours. Note that even though the DF values for uranium are low compared to other sorbates, on a mass basis, uranium loading onto MST is much higher than the other data sets except for the Phase III datasets where the initial uranium and neptunium concentrations were both very high. Because of the relatively high mass concentration of uranium (typically 10,000 ug/L), measureable differences in uranium concentrations are not observable at contact times of 12 hours or less with MST concentrations of 0.2 and $0.4 \mathrm{~g} / \mathrm{L}$ in salt solutions having Na concentrations ranging from 4.5 $\mathrm{M}$ to $7.5 \mathrm{M}$.

For neptunium, most of the DF values measured at $0.2 \mathrm{~g} / \mathrm{L}$ MST were about a factor of 1.3 to 1.5 times lower than that measured at $0.4 \mathrm{~g} / \mathrm{L}$ MST indicating less neptunium removal at the lower MST concentration. The results indicate that at each contact time, the percentage of neptunium removed from solution is about $10 \%$ less upon addition of $0.2 \mathrm{~g} / \mathrm{L}$ MST compared to $0.4 \mathrm{~g} / \mathrm{L}$. This result suggests more effective removal of neptunium at the lower MST concentration, which is confirmed by comparing the calculated loading of neptunium onto to the MST. Neptunium loadings at $0.2 \mathrm{~g} / \mathrm{L}$ MST measured between 1.08 and 2.12 times that at $0.4 \mathrm{~g} / \mathrm{L} \mathrm{MST}$.

The rate of neptunium removal followed the same general trends observed with plutonium. At an MST concentration of $0.2 \mathrm{~g} / \mathrm{L}$, the DF values at a contact time of 4-hours and 8-hours reached on average $85 \%$ and $90 \%$, respectively, of that measured at 12 hours. At an MST concentration of $0.4 \mathrm{~g} / \mathrm{L}$, the DF values at a contact time of 4-hours and 8-hours reached on average $69 \%$ and $83 \%$, respectively, of that measured at 12 hours.

The very large difference in neptunium concentrations across all of the data sets makes it difficult to quantify the influence of ionic strength on the performance of MST to remove neptunium. Inspection of the DF values measured in the Phase III data set revealed no difference in the DF values from the $4.5 \mathrm{M} \mathrm{Na}$ and $7.5 \mathrm{M} \mathrm{Na}$ solutions. Note however, the initial neptunium concentrations in these two solutions varied considerably: 10,500 ug/L in the $4.5 \mathrm{M}$ Na solution and 35,000 ug/L in the 7.5 M Na solution. The Phase IV and Phase V testing featured solutions having nearly identical neptunium concentrations, $406 \mathrm{ug} / \mathrm{L}$ and $403 \mathrm{ug} / \mathrm{L}$, and $\mathrm{Na}$ concentrations of $4.5 \mathrm{M}$ and $5.6 \mathrm{M}$, respectively. Comparison of the DF values in these data sets indicated a small decrease in the DF values at each contact time upon an increase in the Na concentration. It would be expected that the DF values would further decrease in salt solutions having higher $\mathrm{Na}$ concentrations due to the increased competition between $\mathrm{Na}^{+}$and the neptunyl ion, $\mathrm{NpO}_{2}{ }^{+}$for sites on the MST.

Strontium removal in these data sets proceeded to no lower than 94.2\% (DF $=17.1$ ) removal and as high as $99.8 \%(\mathrm{DF}=499)$ removal within 12 hours of contact with MST indicating that MST has high affinity for strontium. In data sets with solutions containing less than $100 \mathrm{ug} / \mathrm{L}$ strontium $>99 \%$ (DF $>100$ ) of the strontium was removed within 4 hours of contact with an MST concentrations of $0.2 \mathrm{~g} / \mathrm{L}$. Even with data sets having salt solutions with strontium concentrations of 1680 and 2,420 ug/L, strontium removal ranged from 94.2\% (DF - 17.1) to 98.6\% (DF = 69.1 with $0.2 \mathrm{~g} / \mathrm{L}$ MST and contact times of 8 hours or less.

As with neptunium, the Phase IV and Phase V datasets featured solutions having nearly identical strontium concentrations, $90.1 \mathrm{ug} / \mathrm{L}$ and $90.5 \mathrm{ug} / \mathrm{L}$, and Na concentrations of $4.5 \mathrm{M}$ and $5.6 \mathrm{M}$, respectively. At each contact time and at both MST concentrations, the DF values decreased with an increase in the Na concentration. For the tests with $0.2 \mathrm{~g} / \mathrm{L} \mathrm{MST}$, the difference was a factor of approximately 4.8. At an MST concentration of $0.4 \mathrm{~g} / \mathrm{L}$ the difference in strontium DF values 
was a factor approximately 3.6. Note, however, that even though the DF values varied by rather large factors, the change in total strontium removed was rather small, $94.2 \%$ versus $99.8 \%$. It would be expected that the DF values would further decrease in salt solutions having higher $\mathrm{Na}$ concentrations due to the increased competition between $\mathrm{Na}^{+}$and $\mathrm{Sr}^{2+}$ for sites on the MST.

Table 3-7 provides a summary of the lowest DF value measured or calculated for each sorbate from each of the data sets reported in the preceding tables at MST concentrations of $0.2 \mathrm{~g} / \mathrm{L}$ and $0.4 \mathrm{~g} / \mathrm{L}$. The standard deviation for the measured values, when available, is also reported. These values may be used to estimate the minimal fraction of each sorbate that would be removed from salt solutions ranging in Na concentrations from 4.5 $\mathrm{M}$ to $7.5 \mathrm{M}$ and given the respective MST concentrations and contact times.

Table 3-7. Minimum DF Values and Single Standard Deviation for Plutonium, Uranium, Neptunium and Strontium in Alkaline Salt Solutions of Varying Ionic Strength Contacted with $0.2 \mathrm{~g} / \mathrm{L}$ or $0.4 \mathrm{~g} / \mathrm{L}$ MST

Average DF Value (Standard Deviation) with 0.2 g/L MST

\begin{tabular}{|c|c|c|c|c|}
\hline Sorbate & $\begin{array}{c}\text { 4-hour } \\
\text { Contact }\end{array}$ & $\begin{array}{c}\text { 6-hour } \\
\text { Contact }\end{array}$ & $\begin{array}{c}\text { 8-hour } \\
\text { Contact }\end{array}$ & $\begin{array}{c}\text { 12-hour } \\
\text { Contact }\end{array}$ \\
\hline $\mathbf{P u}$ & $1.41(0.063)$ & $1.86(0.136)$ & $1.91(0.217)$ & 2.29 \\
\hline $\mathbf{U}$ & $1.02(0)$ & nd & $1.04(0.0147)$ & 1.14 \\
\hline $\mathbf{N p}$ & $1.17(0)$ & $2.55(0)$ & $1.12(0.0193)$ & 1.23 \\
\hline $\mathrm{Sr}$ & $21.7(0.60)$ & $17.1(4.85)$ & 22.0 & $24.8(0.71)$ \\
\hline
\end{tabular}

Average DF Value (Standard Deviation) with 0.4 g/L MST

\begin{tabular}{|c|c|c|c|c|}
\hline Sorbate & $\begin{array}{c}\text { 4-hour } \\
\text { Contact }\end{array}$ & $\begin{array}{c}\text { 6-hour } \\
\text { Contact }\end{array}$ & $\begin{array}{c}\text { 8-hour } \\
\text { Contact }\end{array}$ & $\begin{array}{c}\text { 12-hour } \\
\text { Contact }\end{array}$ \\
\hline Pu & $4.79(0.39)$ & $2.51(0.265)$ & 5.45 & $6.25(0.443)$ \\
\hline $\mathbf{U}$ & $1.04(0.153)$ & $1.06(0.0419)$ & $1.22(0.0817)$ & 1.20 \\
\hline $\mathbf{N p}$ & $1.78(0.272)$ & $1.14(0.0133)$ & 1.33 & $1.40(0)$ \\
\hline $\mathrm{Sr}$ & $48.7(0)$ & $19.6(1.96)$ & 46.0 & $56.8(3.92)$ \\
\hline
\end{tabular}




\subsection{Filtration Performance at Reduced MST Concentration}

The proposed reduction in MST from $0.4 \mathrm{~g} / \mathrm{L}$ to $0.2 \mathrm{~g} / \mathrm{L}$ will affect the performance of the cross flow filter. Models have been used to predict cross flow filter performance as a function of operating parameters. ${ }^{14-16}$ One of the models, the Murkes and Carlsson model, is described by equation [1]

$J=\frac{B \Delta P}{\mu\left[\frac{c^{2} \Delta P}{\left(a v^{3}+b^{3.5}+0.28 v^{4}\right)^{1 / 2}}+L_{0}\right]}$

where $\mathrm{J}$ is filter flux, $\mathrm{B}$ is Darcy's permeability constant for the filter cake, $\Delta \mathrm{P}$ is transmembrane pressure, $\mu$ is liquid viscosity, $v$ is axial velocity, $L_{0}$ is filter resistance, $d_{p}$ is particle size, $c_{v}$ is volumetric concentration of solids, $D$ is filter tube diameter, $\varepsilon$ filter cake porosity, $a=21 \mu / d_{p} \rho_{L}, b$ $=6(\mathrm{a} / 21)^{1 / 2}$, and $\left.\mathrm{c}=2.43 \mathrm{c}_{\mathrm{v}}{ }^{1 / 3}\left[2 \mathrm{D} /(1-\varepsilon) \rho_{\mathrm{L}}\right)\right]^{1 / 2}$. The filter tube resistance, filter cake porosity, and filter tube diameter are assumed to be constant. Using a liquid viscosity of $2.5 \mathrm{cP}$, a particle size of $3 \mu \mathrm{m}$, a liquid density of $1.26 \mathrm{~g} / \mathrm{mL}$, and axial velocities of $10 \mathrm{ft} / \mathrm{s}$ and $15 \mathrm{ft} / \mathrm{s}$, the authors calculated the magnitude of the velocity terms in equation [1], and found the velocity cubed term to be the largest.

The authors simplified equation [1] and produced a new model described by equation [2]

$J=\frac{b_{1} \Delta P v^{1.5}}{\left[b_{2} \Delta P C^{2 / 3} d_{p} \rho_{L}^{2}+\mu v^{1.5}\right\rfloor}$

where $J$ is filter flux $\left(\mathrm{gpm} / \mathrm{ft}^{2}\right), \Delta \mathrm{P}$ is transmembrane pressure (psi), $\mathrm{v}$ is axial velocity $(\mathrm{ft} / \mathrm{s}), \mathrm{C}$ is concentration $(\mathrm{g} / \mathrm{L}), d_{\mathrm{p}}$ is particle size, $\rho_{\mathrm{L}}$ is liquid density, and $\mu$ is liquid viscosity, and $b_{1}$ and $b_{2}$ are constants.

SRNL collected filtration data with simulated SRS waste and full-scale filter tubes (10 ft length, $5 / 8$ inch ID, $0.5 \mu \mathrm{m}$ pore size) and fit the data with the model described by equation [2]. ${ }^{17}$ Since the same feed was used for each of the tests, the particle size, liquid density, and liquid viscosity are assumed to be constant, and equation [2] reduces to

$J=\frac{a_{1} \Delta P v^{1.5}}{\left[a_{2} \Delta P C^{2 / 3}+v^{1.5}\right\rfloor}$

where $\mathrm{a}_{1}$ and $\mathrm{a}_{2}$ are constants.

According to equation [3] filter flux is a function of axial velocity, transmembrane pressure (TMP), and solids concentration. The impact of these factors varies based on the magnitude of each. At high axial velocity, low TMP, or low concentration, the second term in the denominator dominates, and the filter flux is proportional to TMP. At high TMP, high solids concentration, or low axial velocity, the first term in the denominator dominates, and filter flux is a function of axial velocity and solids concentration. At the ARP operating conditions ( $\sim 10 \mathrm{ft} / \mathrm{s}$ axial velocity, $\sim 30 \mathrm{psi}$ TMP, and $0.8-5 \mathrm{wt} \%$ insoluble solids), solids concentration has a significant impact on filter flux.

Figure 3-1 compares the model predictions with data from ARP. The model under-predicts the flux, but the effect of solids concentration is comparable between the model and the data. The model was fit with data from tests with a $0.5 \mu \mathrm{m}$ filter. The ARP uses a $0.1 \mu \mathrm{m}$ filter. SRNL 
testing with actual waste at bench scale and simulated waste at pilot-scale showed the $0.1 \mu \mathrm{m}$ filter produced a larger flux than the $0.5 \mu \mathrm{m}$ filter. ${ }^{18,19}$ The increase ranged from $30 \%$ to $65 \%$. Applying a correction factor of 1.4 to the model predictions shows better agreement with the ARP data. Figure 3-2 compares the predicted flux, using the correction factor, to the ARP operating data. The agreement is good.

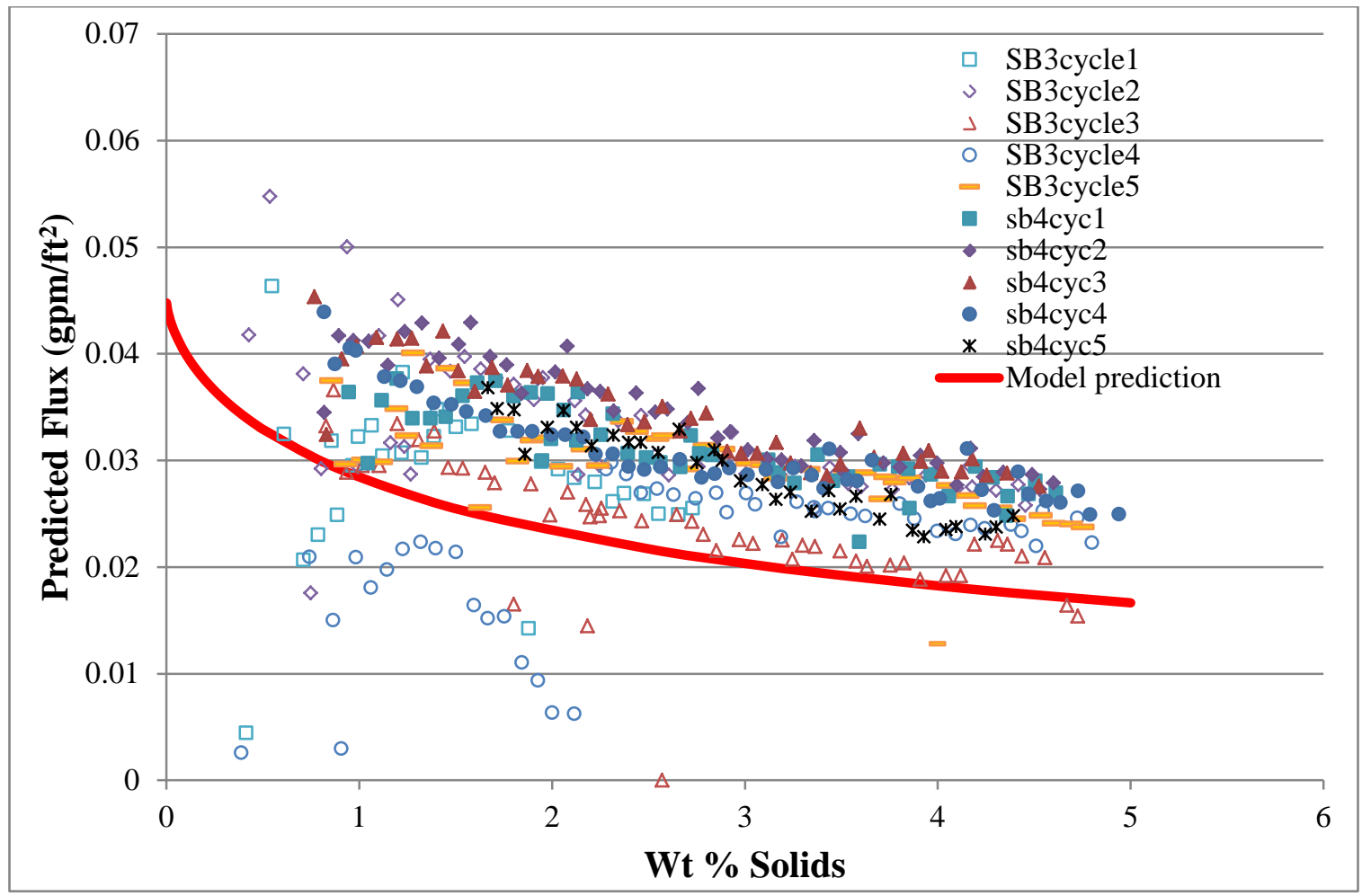

Figure 3-1. Comparing Filter Flux Prediction with ARP Data 


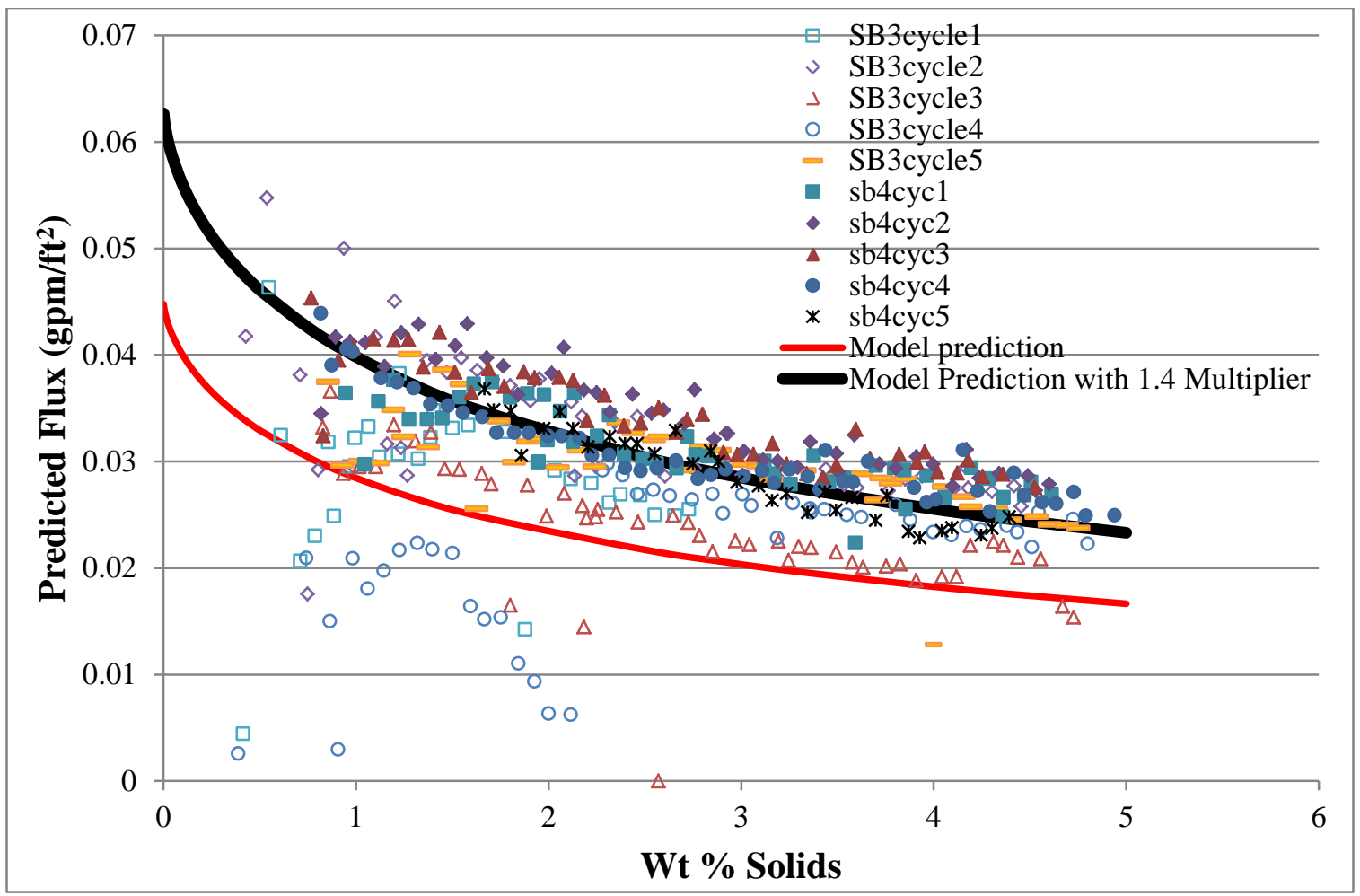

Figure 3-2. Comparing Corrected Filter Flux Prediction with ARP Data

Models are usually fit to the specific system to which they apply. The lack of variation in the axial velocity and TMP at ARP does not lend itself to modeling their effects on filter flux with the ARP data.

By applying the model trend with a multiplier of 1.4, the reduction of MST concentration from $0.4 \mathrm{~g} / \mathrm{L}$ to $0.2 \mathrm{~g} / \mathrm{L}$ is expected to have the following impacts on production. Starting with lower solids will result in an increased filtration rate as predicted by the model. Decreasing from $0.4 \mathrm{~g} / \mathrm{L}$ to $0.2 \mathrm{~g} / \mathrm{L}$ MST is predicted to increase the initial average filtration rate by approximately $10 \%$, or approximately 40 minutes per cycle on average relative to the current performance for the initial cycles. Each cycle will add one-half of the additional solids added per the current batches. Therefore, during operation, the concentration of solids that are fed to the filter will increase more slowly resulting in the filter operating longer at lower solids concentration and the corresponding higher flux.

Thus filter performance is expected to generally follow the prediction shown in Figure 3-2 with filtration rate decreasing as insoluble solids concentration increases during concentration. The filtration rate will decrease and eventually follow the previous production curves but with more feed processed to advance on the concentration curve. The net result will be an initial higher volume of production, eventually matching current production rates. Since other parameters (TMP and axial velocity) are expected to remain consistent with previous operation, the slower increase in solids will result in longer durations at the filtration rates. In other words, the increase in concentration is driving the reduction in flux. Reducing the addition of MST per batch has the net effect of slowing the solids addition rate. That will result in a decrease in flux drop as a function of time, and a higher average filtration rate for each batch. 
Although the model has not been fit to ARP operation, it can be used to estimate of potential impact to process time of reducing the solids loading to a crossflow filter. Using the model to predict the time required to process 50 batches of 3800 gallons of material for the current and reduced MST cases would result in a filtration time savings of up to $20 \%$. This value was obtained by assuming that the only solids are MST; supernate, axial velocity (10.5 ft/sec), and TMP (30 psi) are the same in both cases. Final solids would be an average of $5 \mathrm{wt} \%$ for the 0.4 $\mathrm{g} / \mathrm{L}$ MST case and $2.5 \mathrm{wt} \%$ for the $0.2 \mathrm{~g} / \mathrm{L}$ MST case. The model prediction of a complete cycle with 50 batches is approximately 460 hours of operational time $(0.4 \mathrm{~g} / \mathrm{L}$ MST case). This is comparable to actual data (Salt Batch 3 cycle 2 required 448 hours to process $\sim 190 \mathrm{~K}$ gal in 50 batches).

TMP per the data is approximately 30 psi. Filter theory says that lower filtration rates will result in higher throughputs. At half the filtration rate, the pressure drop will be half as well (Note: at high enough pressure, flux becomes independent of pressure). The filter will operate longer prior to reaching the limiting pressure drop. If cleaning is based on a set schedule (number of batches produced) there may be an opportunity to optimize TMP to increase production rate by reaching a limiting pressure drop at the same time as planned cleaning. One caution is that increased TMP may lead to less efficient cleaning of the filter. This would need to be evaluated. In addition, the expected increase in flux at the initial lower solids may allow for a reduced TMP for those first batches. There may be an opportunity to reduce TMP at low solids though still resulting in a higher flux than historically seen at higher solids. By reducing TMP, the higher production rate could be sustained for a larger volume of product.

Inspection of the data provided from Salt Batches 3 and 4 shows a decrease in TMP during operation. It was concluded that this is due to increased resistance in the guard filter. Ideally, the guard filter and the primary filter should foul at approximately the same rate. This is typically done by adding additional filter area or loading capacity.

\subsection{Conclusions}

TF\&CE is evaluating changes to the ARP operations to decrease the MST concentration to 0.2 $\mathrm{g} / \mathrm{L}$ and contact time to between 6 and 8 hours. For this evaluation, SRNL reviewed previous datasets investigating the performance of MST at $0.2 \mathrm{~g} / \mathrm{L}$ in salt solutions ranging from 4.5 to 7.5 $\mathrm{M}$ in sodium concentration. In general, reducing the MST concentration from 0.4 to $0.2 \mathrm{~g} / \mathrm{L}$ and increasing the ionic strength from 4.5 to $7.5 \mathrm{M}$ in sodium concentration will decrease the measured DF values for plutonium, neptunium, uranium and strontium. Conservatively, low DF values for each sorbate were obtained from a review of the available datasets at contact times of four and eight hours (see Table 3-3). These DF values are applicable within the sorbate and sodium concentrations used in the experimental measurements.

Decreasing the MST concentration in the ARP from $0.4 \mathrm{~g} / \mathrm{L}$ to $0.2 \mathrm{~g} / \mathrm{L}$ will produce an increase in the filter flux, and could lead to longer operating times between filter cleaning. The increase in flux is a function of a number of operating parameters, and is difficult to quantify. A general estimation has been made based on filtration theory of up to a $20 \%$ increase in filtration time as a result of decreasing MST solids.

\subsection{Recommendations, Path Forward or Future Work}

Evaluate if increased TMP leads to less efficient cleaning of the filter. 


\subsection{Acknowledgements}

The authors thank T. B. Peters for calculating the sorbate concentrations in mass concentration units for ARP/MCU Macrobatches $1-5$.

\subsection{References}

1. Lang, K. L., Technical Task Request X-TTR-H-00018, Revision 0, MST Reduction Analysis for ARP/MCU, April 18, 2012.

2. Adsorption of Actinides in MST Strike Tank 1 and Strike Tank 2, 96H Actinide Removal Process Manual, SW9.2-IOP-96H, Section 4.2 and Section 4.3, Rev. 6, February 3, 2011.

3. Nash, C.A.; Peters, T.B.; Fink, S.D., "Tank 49H Salt Batch Supernate Qualification for ARP/MCU”, WSRC-STI-2008-00117, Rev. 0, August 25, 2008.

4. Peters, T.B.; Nash, C.A.; Fink, S.D., "ISDP Salt Batch \#2 Supernate Qualification”, SRNL-STI-200800446, Rev. 1, January 5, 2009.

5. Peters, T.B.; Fink, S.D., "ISDP Salt Batch \#2 MST Tests Using Reduced Quantities of MST”, SRNLSTI-2009-00063, Rev. 0, March 6, 2009.

6. Peters, T.B.; Fink, S.D., "Results from Monosodium Titanate (MST) and Extraction-Scrub-Strip (ESS) Testing of ISDP Macrobatch 3 Blend”, SRNL-STI-2010-00290, Rev. 0, May, 2010.

7. Peters, T.B.; Fink, S.D., "Sample Results from the Integrated Salt Disposition Program Macrobatch 4 Tank 21H Qualification Samples”, SRNL-STI-2011-00061, Rev. 0, June 2011.

8. Peters, T.B.; Fink, S.D., "Sample Results from the Integrated Salt Disposition Program Macrobatch 5 Tank 21H Qualification Samples”, SRNL-STI-2012-00207, Rev. 0, April 2012.

9. Hobbs, D.T.; Bronikowski, M.G.; Edwards, T.B.; Pulmano, R.L., "Final Report on Phase III Testing of Monosodium Titanate Adsorption Kinetics", Technical Report WSRC-TR-99-00134, Rev. 0, Westinghouse Savannah River Company, Aiken, SC, May 28, 1999.

10. Hobbs, D.T.; Blume, M.S.; Thacker, H.L., "Phase V Simulant Testing of Monosodium Titanate Adsorption Kinetics”, Technical Report WSRC-TR-2000-00142, Rev. 0, Westinghouse Savannah River Company, Aiken, SC, May 24, 2000.

11. Hobbs, D.T.; Nyman, M.L.; Peters, T.B.; Poirier, M.R.; Barnes, M.J.; Thompson, M.E.; Fink, S.D., "Tailoring Inorganic Sorbents for SRS Strontium and Actinide Separations: Optimized Monosodium Titanate Phase II Final Report”, Technical Report WSRC-TR-2007-00082, Rev. 0, Westinghouse Savannah River Company, Aiken, SC, June 2007.

12. Hobbs, D.T.; Pulmano, R.L., "Phase IV Simulant Testing of Monosodium Titanate Adsorption Kinetics”, Technical Report WSRC-TR-99-00219, Rev. 0, Westinghouse Savannah River Company, Aiken, SC, June 29, 1999.

13. Taylor-Pashow, K.M.L.; Nash, C.A.; Hobbs, D.T., "Testing and Characterization of Engineered Forms of Monosodium Titanate (MST)”, Technical Report SRNL-STI-2012-00193, Rev. 0, Savannah River Nuclear Solutions LLC, Aiken, SC, May 2012.

14. Peterson, R. A., and Nash, C. A., "Filter Performance Mechanisms", WSRC-TR-95-0420, Westinghouse Savannah River Company, Aiken, SC, October 20, 1995.

15. Porter, M. C. “Concentration Polarization with Membrane Ultrafiltration”, Ind. Eng. Chem. Prod. Res. Develop., vol. 11, No. 3, 1972, pp. 234-248.

16. Jakob Murkes and Claes-Goran Carlsson, Crossflow Filtration: Theory and Practice, New York: Wiley, 1988.

17. Poirier, M. R., Fink, S. D., Henry, P. Haggard, R.., Deal, T. and Van Brunt, V., "Modeling of PilotScale Cross-Flow Filtration of Simulated Nuclear Waste", Technical Report WSRC-MS-03-00830, Westinghouse Savannah River Company, Aiken, SC, November 20, 2003.

18. Poirier, M. R. and Fink, S. D., "Recommendation for using Smaller $(0.1 \mu)$ Pore Size Media for Filtration in Salt Waste Processing Project”, Technical Report WSRC-TR-2002-00341, Westinghouse Savannah River Company, Aiken, SC, August 5, 2002.

19. Poirier, M. R., Siler, J. L., Fink, S. D., Haggard, R., Stork, C. and Van Brunt, V., "Pilot-Scale Testing of a $0.1 \mu$ Filter with Simulated SRS High Level Waste”, Technical Report WSRC-TR-2003-00469, Westinghouse Savannah River Company, Aiken, SC, October 8, 2003. 


\section{Appendix}

Figure A-1. Plutonium DF values versus time from salt solutions of varying sodium concentration. MST concentration equals $0.2 \mathrm{~g} / \mathrm{L}$ (upper) and $0.4 \mathrm{~g} / \mathrm{L}$ (lower).
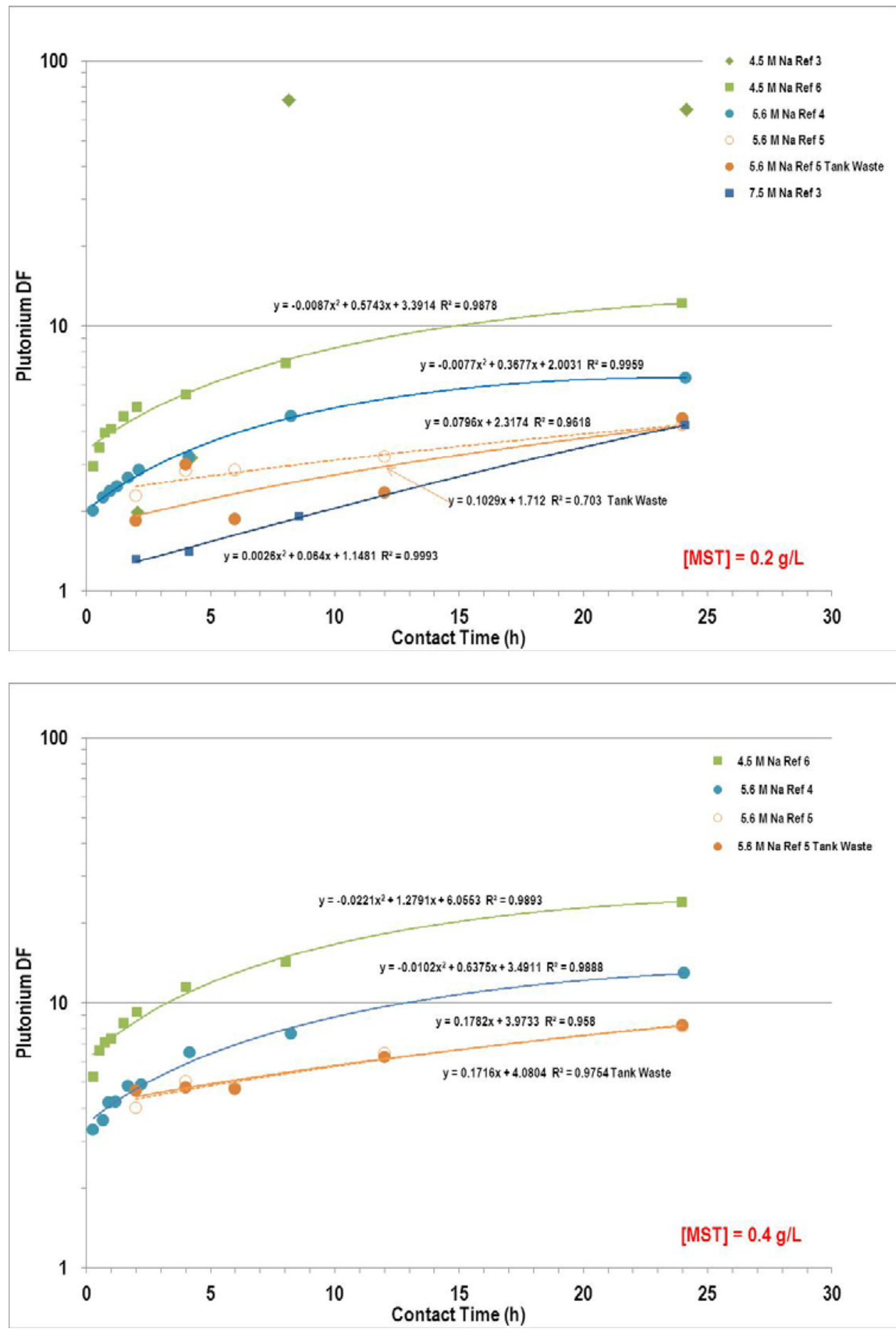
Figure A-2. Uranium DF values versus time from salt solutions of varying sodium concentration. MST concentration equals $0.2 \mathrm{~g} / \mathrm{L}$ (upper) and $0.4 \mathrm{~g} / \mathrm{L}$ (lower).
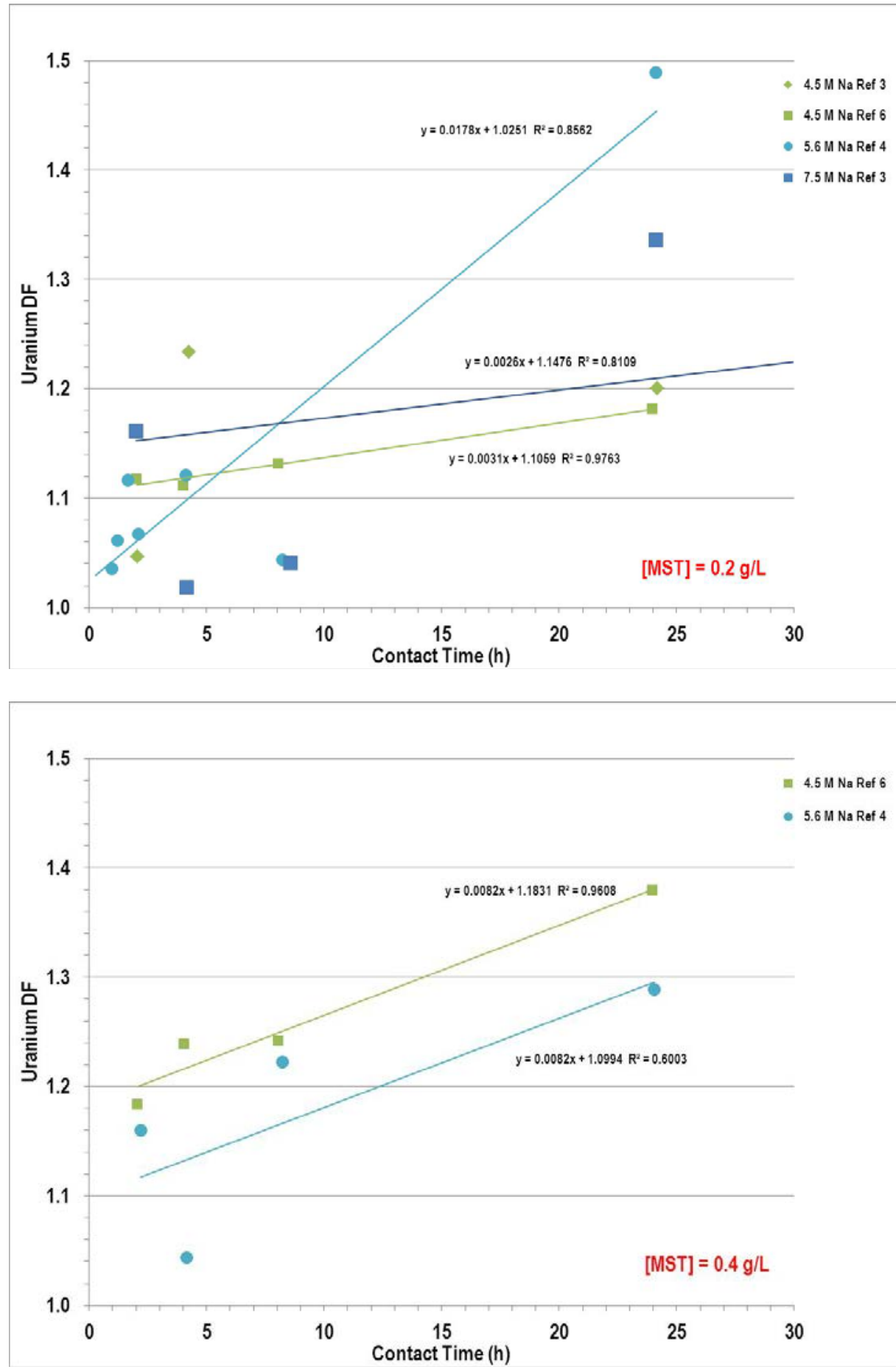
Figure A-3. Neptunium DF values versus time from salt solutions of varying sodium concentration. MST concentration equals $0.2 \mathrm{~g} / \mathrm{L}$ (upper) and $0.4 \mathrm{~g} / \mathrm{L}$ (lower).
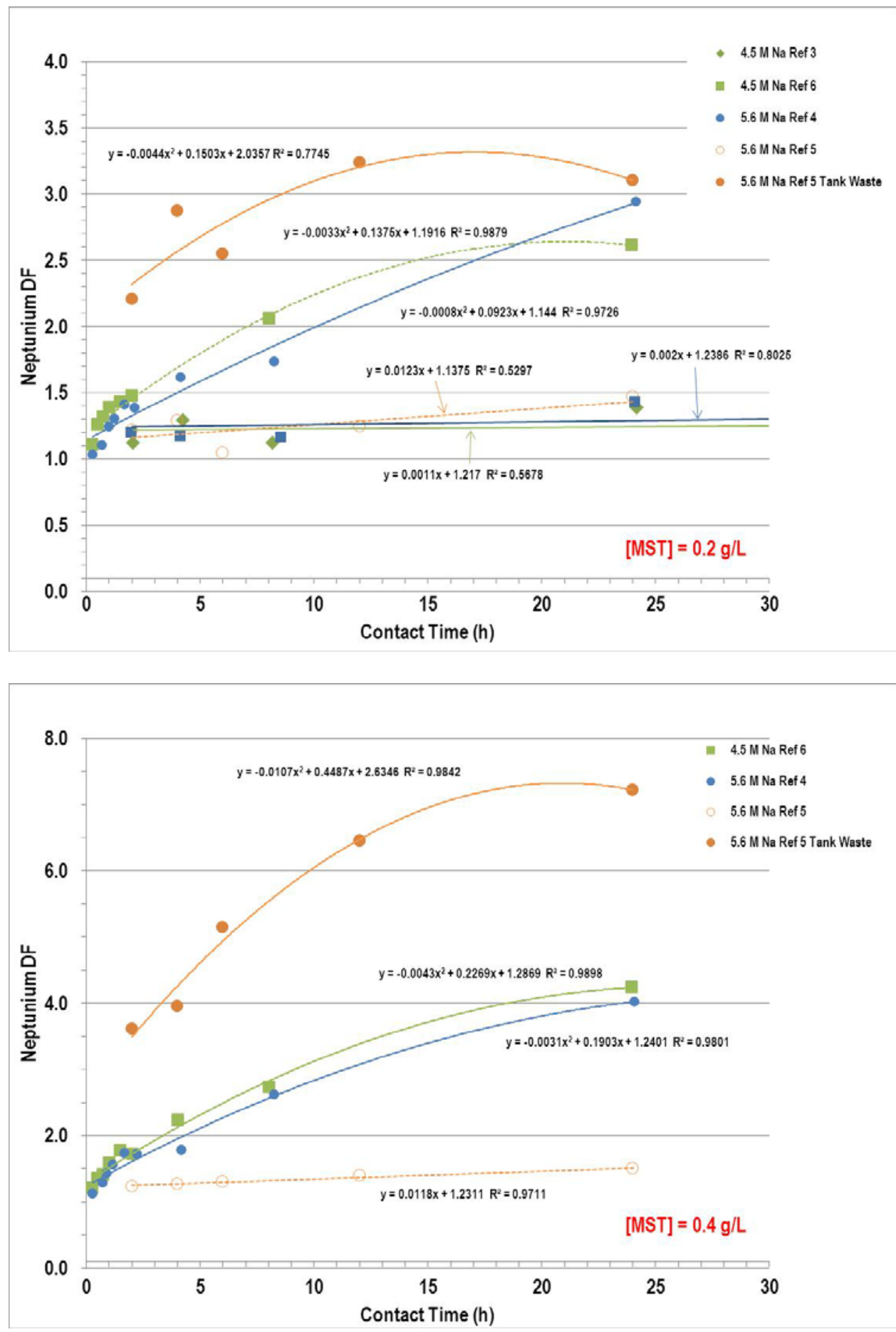
Figure A-4. Strontium DF values versus time from salt solutions of varying sodium concentration. MST concentration equals $0.2 \mathrm{~g} / \mathrm{L}$ (upper) and $0.4 \mathrm{~g} / \mathrm{L}$ (lower).
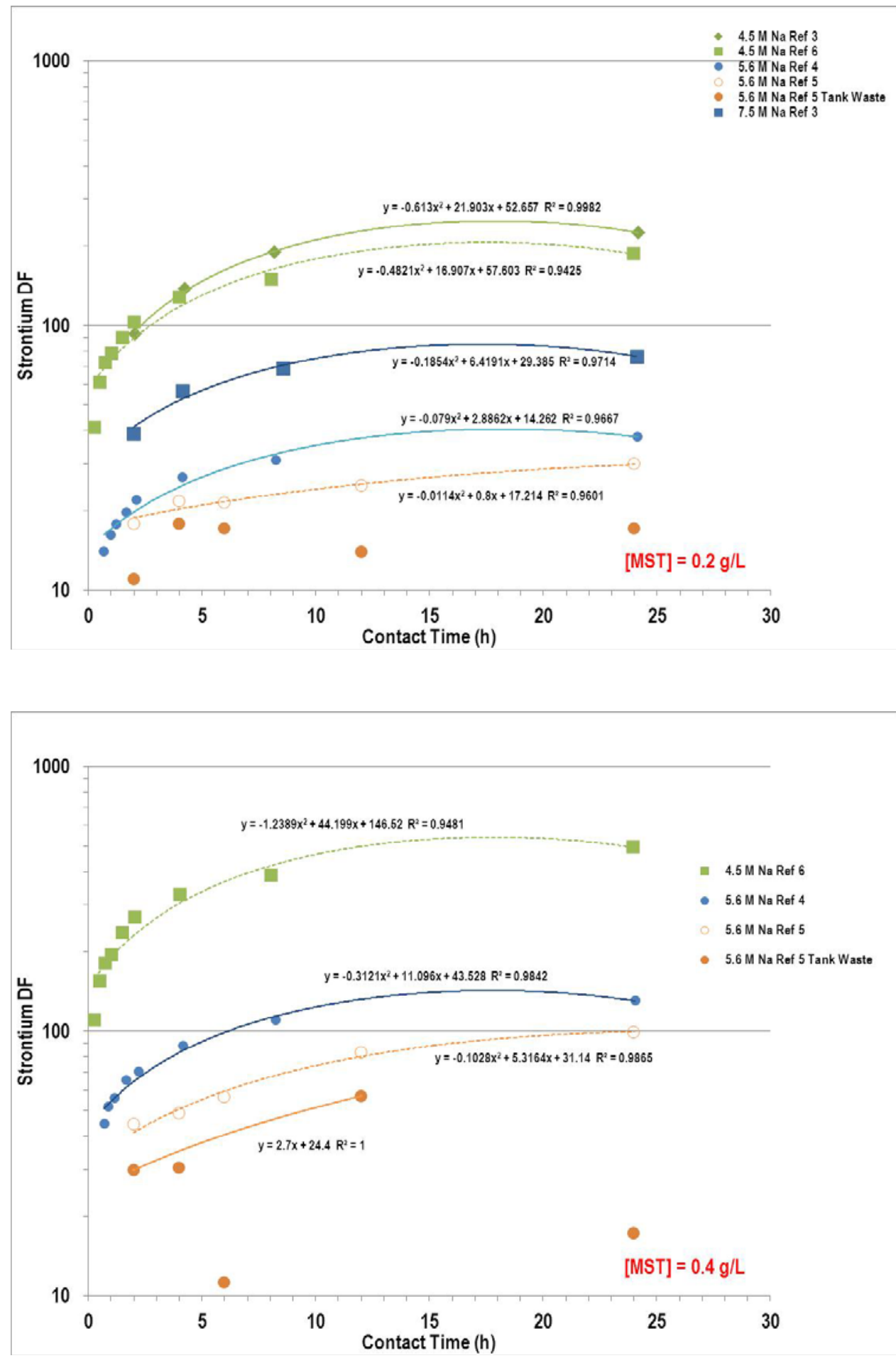


\section{Distribution:}

S. D. Fink, 773-A

K. M. Fox, 999-W

B. J. Giddings, 786-5A

C. C. Herman, 999-W

S. L. Marra, 773-A

F. M. Pennebaker, 773-42A

D.T. Hobbs, 773-A

D. T. Herman, 735-11A

M. R. Poirier, 773-42A

C. Wilson, 773-A

Records Administration (EDWS)

E. J. Freed, 704-56H

D. J. Martin, 241-152H

K. L. Lang, 704-27S

R. V. Doniphan, 241-156H

M. W. Geeting, 241-152H

C. J. Scherman, 241-197H

M. H. Barnett, 766-H

T. A. Le, 766-H

A. R. Shafer, 704-27S

C. K. Chiu, 704-27S

S. E. Campbell, 241-152H

S. P. Mcleskey, 241-152H

B. A. Gifford, 704-56H

R. M. Wolfenden, 704-56 H

K. H. Subramanian, 766-H

K. D. Harp, 766-H

R. K. Leugemors, 992-5W

W. B. Brasel, 992-2W

P. R. Jackson, DOE-SR, 703-46A 